\section{REGISTRO DE PACIENTES CON INMUNODEFICIENCIAS PRIMARIAS EN LOS TRES PRINCIPALES CENTROS DE REFERENCIA DEL PERÚ}

\author{
REGISTRATION OF PATIENTS WITH PRIMARY \\ IMMUNODEFICIENCIES IN THE THREE MAIN \\ REFERENCE CENTERS IN PERU
}

\author{
David García-Gomero 1,2,3,a, Wilmer Córdova-Calderón 1,3,4,b, \\ Juan Aldave-Becerra ${ }^{1,3,5, b}$
}

Sr. Editor. Las inmunodeficiencias primarias (IDP) incluyen un conjunto de trastornos hereditarios de la función del sistema inmunitario ${ }^{(1)}$. Según clasificación de la International Union of Immunological Societies (IUIS) estos trastornos se pueden dividir en nueve categorías: inmunodeficiencias combinadas, deficiencias predominantemente de anticuerpos, inmunodeficiencias combinadas con síndromes bien definidos, enfermedades de desregulación inmune, defectos congénitos del número, función o ambos del sistema fagocítico; defectos de la inmunidad innata, desordenes autoinflamatorios, deficiencias del complemento e inmunodeficiencias por fenocopias ${ }^{(2)}$.

Existen signos de sospecha de IDP establecidos por la Jeffrey Modell Foundation (JMF) que se basan en la aparición de infecciones recurrentes en diferentes aparatos y sistemas ${ }^{(1)}$. La Latin American Society for Immunodeficiencies (LASID) creó un sistema de registro para los casos de IDP ${ }^{(3)}$. En el Perú, existen tres centros de referencia públicos que contribuyen con LASID en el registro de los pacientes con IDP: el Centro de Referencia Nacional de Alergia, Asma e Inmunología (CERNAAI) del Instituto Nacional de Salud del Niño (INSN), el Hospital Nacional Edgardo Rebagliati Martins (HNERM) y el Hospital Nacional Guillermo Almenara Irigoyen (HNGAI). Pretendemos realizar un reporte del número de casos y algunas características de los pacientes con IDP atendidos en estos centros.

\footnotetext{
1 Centro de Referencia Nacional de Alergia, Asma e Inmunología. Instituto Nacional de Salud del Niño. Lima, Perú.

2 Facultad de Medicina. Universidad Nacional Mayor de San Marcos. Lima, Perú.

Sociedad Peruana de Inmunología. Lima, Perú.

4 Grupo peruano de Inmunodeficiencias Primarias (PERUGID). Lima, Perú.

Servicio de Alergias e Inmunología. Hospital Nacional Edgardo Rebagliati Martins. Lima, Perú.

a Médico cirujano, ${ }^{\mathrm{b}}$ Médico inmunólogo clínico-alergólogo.

Recibido: 21/12/2017 Aprobado: 17/01/2018 En línea:29/08/2018
}

Citar como: García-Gomero D, Córdova-Calderón W, Aldave-Becerra J. Registro de pacientes con inmunodeficiencias primarias en los tres principales centros de referencia del Perú. Rev Peru Med Exp Salud Publica. 2018;35(3): 53840. doi: 10.17843/rpmesp.2018.353.3317.
Se hizo revisión de los registros anónimos de los pacientes atendidos entre agosto del 2015 y abril del 2017, previamente se obtuvo la autorización de las instancias pertinentes en cada centro. Se obtuvieron datos como edad, género, institución, tipo de seguro, diagnóstico y grupo al cual pertenece según IUIS. Se incluyeron a 116 pacientes menores de 18 años y 16 con 18 años o más. La edad mediana fue 6 (rango intercuartil: 0,0515) años para los pacientes menores de 18 años y 47,5 (rango intercuartil: 20-54) para los de 18 años o más; 91/132 (68\%) fueron varones. Para las categorías de la IUIS, las deficiencias predominantemente de anticuerpos se hallan en 39,4\% (52/132) de los pacientes, de estos 32,7 \% (17/52) corresponden a agammaglobulinemias primarias y $30,7 \%$ (16/52) corresponden a agammaglobulinemias no especificadas.

La inmunodeficiencia común variable se halla en $21,2 \%$ (11/132), los defectos congénitos del número, función o ambos del sistema fagocitario los encontramos en $16,7 \%$ (22/132); entre ellas se encontró neutropenia congénita en $54,5 \%(12 / 22)$, enfermedad granulomatosa crónica en $31,8 \%$ y deficiencia de adhesión leucocitaria en 13,6\% (3/22). Las inmunodeficiencias combinadas con otros síndromes bien definidos fueron encontradas en 13,6 \% (18/132) de los pacientes, entre ellos se identificaron el Síndrome Hiper IgE en 44,4 \% (8/18), a la Ataxia Telangiectasia en $27,8 \%$ (5/18) y al Síndrome de Di George en 11,1 \% (2/18).

Las inmunodeficiencias que afectan la inmunidad celular y humoral afectaron al 3,0 \% (4/132) de pacientes; las enfermedades por disregulación inmune afectaron al 3,0 \% (4/132); al igual que los defectos de la inmunidad innata $(3,0 \%, 4 / 132)$ y los defectos del complemento afectado al $1,5 \%(2 / 132)$ pacientes. Finalmente encontramos que $16,7 \%(22 / 132)$ pacientes cumplen con los criterios de la Jeffrey Model Foundation (JMF), pero aún se hallan en estudios. No encontramos pacientes con síndromes autoinflamatorios, ni Inmunodeficiencias por fenocopias. (Tabla 1).

Se puede concluir que la mayor parte de los pacientes acuden a establecimientos del seguro social (EsSalud) y que, tal como se describe en la literatura ${ }^{(5)}$, los defectos en la producción de anticuerpos son la categoría predominante $y$, dentro de ellos, las agammaglobulinemias primarias. Por último, ya que la mayor parte de pacientes se halla en espera de un diagnóstico específico, se requiere implementar la logística necesaria en los centros hospitalarios para brindar un diagnóstico y tratamiento tempranos.

Fuentes de financiamiento: Grupo peruano de inmunodeficiencias primarias-PERUGID.

\section{Conflictos de interés: Ninguno}

Contribuciones de autoría: DGG y WCC han participado en la concepción del artículo. JAB y WC C participaron en la recolección de datos. DGG se encargó del procesamiento, análisis de datos y redacción del artículo. JAB y WC participaron en la revisión crítica del artículo y aprobación de la versión final. WCC obtuvo el financiamiento. 
Tabla 1. Descripción de 132 pacientes peruanos con Inmunodeficiencias Primarias según la clasificación de la International Union of Immunological Societies. Lima-Perú, 2017.

\begin{tabular}{|c|c|c|c|c|c|c|}
\hline \multirow[t]{2}{*}{ Variables } & \multicolumn{2}{|c|}{$\begin{array}{l}0 \text { a } 17 \text { años } \\
(n=116)\end{array}$} & \multicolumn{2}{|c|}{$\begin{array}{c}18 \text { años a más } \\
(n=16)\end{array}$} & \multicolumn{2}{|c|}{$\begin{array}{c}\text { Total } \\
(n=132)\end{array}$} \\
\hline & $\mathbf{n}$ & $\%$ & $\mathbf{n}$ & $\%$ & $\mathbf{n}$ & $\%$ \\
\hline \multicolumn{7}{|l|}{ Aspectos sociodemográficos } \\
\hline Edad mediana (rango intercuartil) & 6 & $(0,5-15)$ & 39,5 & $(20-54)$ & 7 & $(0,5-54)$ \\
\hline \multicolumn{7}{|l|}{ Sexo } \\
\hline Masculino & 82 & 70,7 & 9 & 56,3 & 91 & 68,9 \\
\hline Femenino & 34 & 29,3 & 7 & 43,8 & 41 & 31,1 \\
\hline \multicolumn{7}{|l|}{ Hospital de origen } \\
\hline Instituto Nacional de Salud del Niño & 52 & 44,8 & 1 & 6,3 & 53 & 40,2 \\
\hline Edgardo Rebagliati Martins & 36 & 31,0 & 0 & 0 & 36 & 27,3 \\
\hline Guillermo Almenara Irigoyen & 19 & 16,4 & 15 & 93,8 & 34 & 25,8 \\
\hline Otro hospital EsSalud & 3 & 2,6 & 0 & 0 & 3 & 2,3 \\
\hline María Auxiliadora & 2 & 1,7 & 0 & 0 & 2 & 1,5 \\
\hline Arzobispo Loayza & 1 & 0,9 & 0 & 0 & 1 & 0,8 \\
\hline Daniel Alcides Carrión & 1 & 0,9 & 0 & 0 & 1 & 0,8 \\
\hline Luis N, Sáenz de la Policía Nacional del Perú & 1 & 0,9 & 0 & 0 & 1 & 0,8 \\
\hline Hospital Regional Lambayeque & 1 & 0,9 & 0 & 0 & 1 & 0,8 \\
\hline \multicolumn{7}{|l|}{ Institución donde se atiende actualmente } \\
\hline Instituto Nacional de Salud del Niño & 61 & 52,6 & 1 & 6,2 & 62 & 47,0 \\
\hline Edgardo Rebagliati Martins & 36 & 31,0 & 0 & 0 & 36 & 27,2 \\
\hline Guillermo Almenara Irigoyen & 19 & 16,4 & 15 & 93,8 & 34 & 25,8 \\
\hline \multicolumn{7}{|l|}{ Tipo de seguro n (\%) } \\
\hline EsSalud & 58 & 50,0 & 15 & 93,8 & 73 & 55,3 \\
\hline Seguro Integral de Salud & 57 & 49,1 & 1 & 6,3 & 58 & 43,9 \\
\hline Sanidad de la Policía Nacional del Perú & 1 & 0,9 & 0 & 0 & 1 & 0,8 \\
\hline \multicolumn{7}{|l|}{ Descripción según la clasificación IUIS** } \\
\hline Deficiencias predominantemente de anticuerpos & 42 & 36,2 & 10 & 62,5 & 52 & 39,4 \\
\hline Agammaglobulinemia primaria & 14 & 12,1 & 3 & 18,8 & 17 & 12,9 \\
\hline Agammaglobulinemia no especificada & 16 & 13,8 & 0 & 0 & 16 & 12,1 \\
\hline Inmunodeficiencia común variable & 7 & 6,0 & 4 & 25,0 & 11 & 8,3 \\
\hline Síndrome Híper lgM & 3 & 2,6 & 3 & 18,8 & 6 & 4,5 \\
\hline Deficiencia selectiva de $\lg A$ & 1 & 0,9 & 0 & 0 & 1 & 0,8 \\
\hline $\begin{array}{l}\text { Deficiencias de isotipo o cadena ligera con un número generalmente } \\
\text { normal de células B }\end{array}$ & 1 & 0,9 & 0 & 0 & 1 & 0,8 \\
\hline Defectos Congénitos de número, función o ambos del sistema Fagocitario & 21 & 18,1 & 1 & 6,3 & 22 & 16,7 \\
\hline Neutropenia congénita & 12 & 10,3 & 0 & 0 & 12 & 9,1 \\
\hline Enfermedad granulomatosa crónica & 6 & 5,2 & 1 & 6,3 & 7 & 5,3 \\
\hline Deficiencia de adhesión leucocitaria & 3 & 2,6 & 0 & 0 & 3 & 2,3 \\
\hline Inmunodeficiencias combinadas con otros síndromes bien definidos & 14 & 12,1 & 3 & 18,7 & 17 & 12,9 \\
\hline Síndrome Híper lgE & 5 & 4,3 & 3 & 18,8 & 8 & 6,1 \\
\hline Defecto de reparación de DNA & 5 & 4,3 & 0 & 0 & 5 & 3,8 \\
\hline Defectos tímicos con anomalías congénitas (Síndrome de Di George) & 2 & 1,7 & 0 & 0 & 2 & 1,5 \\
\hline Infecciones recurrentes + autoinmunidad & 2 & 1,7 & 0 & 0 & 2 & 1,5 \\
\hline Inmunodeficiencias que afectan a la inmunidad celular y humoral & 5 & 4,3 & 0 & 0 & 5 & 3,8 \\
\hline Inmunodeficiencia combinada & 2 & 1,7 & 0 & 0 & 2 & 1,5 \\
\hline Deficiencia de ZAP70 & 1 & 0,9 & 0 & 0 & 1 & 0,8 \\
\hline Deficiencia linfocitos CD4 específica & 1 & 0,9 & 0 & 0 & 1 & 0,8 \\
\hline Enfermedades por disregulación inmune & 4 & 3,4 & 0 & 0 & 4 & 3,0 \\
\hline Síndrome hemofagocítico & 2 & 1,7 & 0 & 0 & 2 & 1,5 \\
\hline Síndrome linfoproliferativo ligado al Cromosoma X & 2 & 1,7 & 0 & 0 & 2 & 1,5 \\
\hline Síndrome linfoproliferativo autoinmune probable, & 1 & 0,9 & 0 & 0 & 1 & 0,8 \\
\hline Defectos de la inmunidad innata & 4 & 3,4 & 0 & 0 & 4 & 3,0 \\
\hline Candidiasis mucocutánea crónica & 2 & 1,7 & 0 & 0 & 2 & 1,5 \\
\hline Susceptibilidad mendeliana a micobacterias & 2 & 1,7 & 0 & 0 & 2 & 1,5 \\
\hline Defectos del complemento & 0 & 0 & 2 & 12,5 & 2 & 1,5 \\
\hline Deficiencia de C1 inhibidor & 0 & 0 & 2 & 12,5 & 2 & 1,5 \\
\hline Cumple criterios $\mathrm{JMF}^{*}$, pero aún en estudios & 23 & 19,8 & 0 & 0 & 23 & 17,4 \\
\hline Inmunodeficiencia sin especificar & 23 & 19,8 & 0 & 0 & 23 & 17,4 \\
\hline
\end{tabular}

*Jeffrey Modell Foundation

** International Union of Immunological Societies 


\section{REFERENCIAS BIBLIOGRÁFICAS}

1. Picard C, Al W, Bousfiha A, Casanova J, Chatila T, Conley M, et al. Primary Immunodeficiency Diseases: an Update on the Classification from the International Union of Immunological Societies Expert Committee for Primary Immunodeficiency 2015. J Clin Immunol. 2015;35(8):696-726.

2. Bousfiha A, Jeddane L, Al-Herz W, Ailal F, Casanova J, Chatila T, et al. The 2015 IUIS Phenotypic Classification for Primary Immunodeficiencies. J Clin Immunol. 2015;35(8):727-38.

3. Costa B, González M, Espinosa S, Segundo G. Latin American challenges with the diagnosis and treatment of primary immunodeficiency diseases. Expert Rev Clin Immunol. 2016; 21:1-7.

4. Modell V, Quinn J, Ginsberg G, Gladue R, Orange J. Jeffrey Modell Foundation modeling strategy to identify patients with primary immunodeficiency utilizing risk management and outcome measurement. Immunol Res. 2017;65(3):713-20.

5. Bonilla F, Khan D, Ballas Z, Chinen J, Frank M, Hsu J, et al. Practice parameter for the diagnosis and management of primary immunodeficiency. J Allergy Clin Immunol. 2015;136(5):1186-1205.

Correspondencia: David Santiago García Gomero.

Dirección: Calle Quipán 157 Urb. Tahuantinsuyo-Independencia.

Teléfono: 977325248

Correo electrónico:dsantg.g@gmail.com

\section{SOBRE LA EVALUACIÓN DEL TRASTORNO POR ESTRÉS POSTRAUMÁTICO A LOS AFECTADOS POR EL NIÑO}

\section{ON THE EVALUATION OF POST-TRAUMATIC STRESS DISORDER IN INDIVIDUALS AFFECTED BY EL NIÑO}

\section{Sergio Dominguez-Lara ${ }^{1, a}$}

Sr. Editor. Sin duda, la comprensión del estado emocional de las víctimas de fenómenos naturales puede brindar información relevante a fin de orientar las intervenciones según la gravedad de las mismas. Sin embargo, cuando los datos presentados se basan en puntuaciones de instrumentos de uso cuestionable, el costo podría ser aún mayor.

En un estudio reciente de Espinoza-Neyra y colegas ${ }^{(1)}$ se reporta la prevalencia del trastorno por estrés postraumático

\footnotetext{
1 Facultad de Ciencias de la Comunicación, Turismo y Psicología. Universidad de San Martín de Porres. Lima, Perú

a Psicólogo, Magíster en Psicología.

Recibido: 09/02/2018 Aprobado: 21/02/2018 En línea: 28/09/2018
}

Citar como: Dominguez-Lara S. Sobre la evaluación del trastorno por estrés postraumático a los afectados por El Niño. Rev Peru Med Exp Salud Publica. 2018;35(3):540.doi: 10.17843/rpmesp.2018.353.3467.
(TEPT) en personas afectadas por el niño costero, y aunque dada su magnitud este puede percibirse como grave, existen tres puntos a tomar en cuenta.

El primero, que la escala de trauma de Davidson es un instrumento que no cuenta con estudios psicométricos publicados con muestras peruanas que avalen sus bondades psicométricas, lo que incluye la adecuación lingüística al contexto donde se aplicó (o al menos, no es mencionado), así como el análisis de su dimensionalidad (¿es una medida unidimensional o multidimensional?) y la confiabilidad de sus puntuaciones.

El segundo punto está vinculado con la confiabilidad, y es que no queda claro qué puntos de corte fueron utilizados para clasificar a las personas en con y sin TEPT, considerando que este es el aspecto medular del trabajo. El tercer punto, es que el instrumento emplea como base al DSM-IV, mientras que el 2016 fue publicada la versión revisada ${ }^{(2)}$, que se ajusta más al DSM-V. El estudio más reciente (2) provee de forma ordenada todos los procedimientos que podrían ser empleados en un potencial estudio psicométrico del instrumento revisado en diversas muestras de interés.

En conclusión, ante la ausencia de información psicométrica relevante a este contexto y que no se encuentra en sintonía con el DSM-V, la escala de trauma de Davidson no reúne los requisitos necesarios para considerar la interpretación de sus puntuaciones como válida ${ }^{(3)}$. Esto podría traer como corolario una clasificación inadecuada de los examinados, y si bien el diagnóstico no se basa sólo en un autoinforme, este es usado como herramienta de apoyo para esa labor.

Fuentes de financiamiento: Autofinanciado

Conflicto de intereses: El autor declara no tener ningún conflicto de intereses.

\section{REFERENCIAS BIBLIOGRÁFICAS}

1. Espinoza-Neyra C, Jara-Pereda M, Díaz-Vélez C.Trastorno de estrés postraumático en una población afectada por inundaciones ocasionadas por el niño costero en Perú. Rev Peru Med Exp Salud Pública. 2017;34(4):751-2.

2. Echeburúa E, Amor PJ, Sarasua B, Zubizarreta I, Holgado-Tello FP, Muñoz JM. Escala de Gravedad de Síntomas Revisada (EGS-R) del Trastorno de Estrés Postraumático según el DSM-5: propiedades psicométricas. Terapia Psicológica 2016; 34(2): 111-28.

3. American Educational Research Association, American Psychological Association, National Council on Measurement in Education [AERA/APA/NCME]. Standards for educational and psychological testing. Washington, DC: American Psychological Association; 2014

Correspondencia: Sergio Dominguez-Lara

Dirección: Instituto de Investigación de Psicologia, Universidad de San

Martín de Porres, Av. Tomás Marsano 242 (5to piso), Lima 34 - Perú.

Correo electrónico:sdominguezl@usmp.pe 